\title{
Countability of Inductive Types Formalized in the Object-Logic Level
}

\author{
Qinxiang Cao Xiwei $\mathrm{Wu}^{*}$
}

\begin{abstract}
The set of integer number lists with finite length, and the set of binary trees with integer labels are both countably infinite. Many inductively defined types also have countably many elements. In this paper, we formalize the syntax of first order inductive definitions in Coq and prove them countable, under some side conditions. Instead of writing a proof generator in a meta language, we develop an axiom-free proof in the Coq object logic. In other words, our proof is a dependently typed Coq function from the syntax of the inductive definition to the countability of the type. Based on this proof, we provide a Coq tactic to automatically prove the countability of concrete inductive types. We also developed Coq libraries for countability and for the syntax of inductive definitions, which have value on their own.
\end{abstract}

Keywords: countable, Coq, dependent type, inductive type, object logic, meta logic

\section{Introduction}

In type theory, a system supports inductive types if it allows users to define new types from constants and functions that create terms of objects of that type. The Calculus of Inductive Constructions (CIC) is a powerful language that aims to represent both functional programs in the style of the ML language and proofs in higher-order logic [16]. Its extensions are used as the kernel language of Coq [5] and Lean [14], both of which are widely used, and are widely considered to be a great success. In this paper, we focus on a common property, countability, of all first-order inductive types, and provide a general proof in Coq's object-logic. The techniques that we use in this formalization can be useful for formally proving other properties of inductive types in the future.

Here we show some examples of inductive types that we will use in this paper :

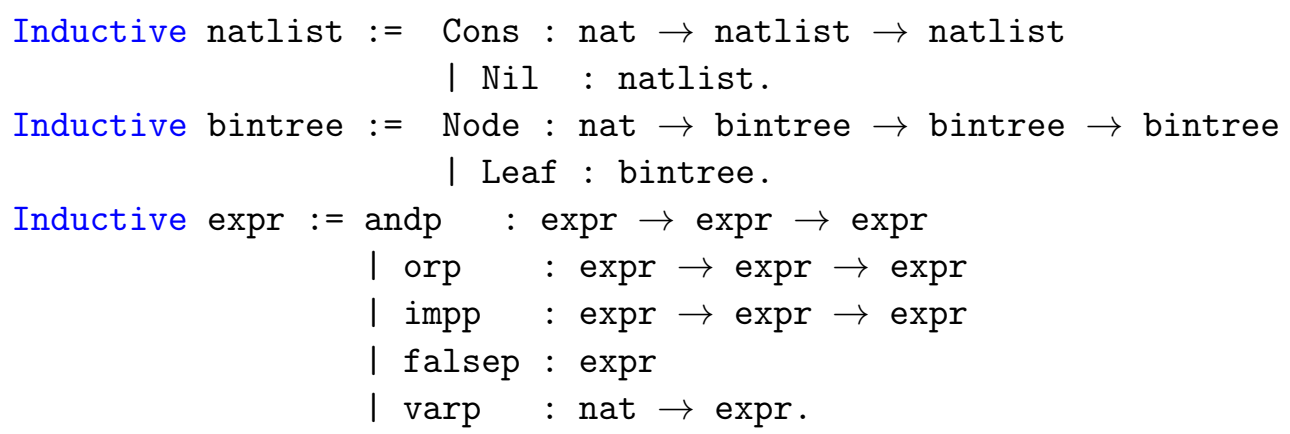

As demonstrated above, natlist (list of natural numbers), bintree (binary trees with natural numbers as labels) and expr (the expressions of propositional language with nat as variable identifiers) can be defined inductively in the Coq proof assistant [5]. Here, the word "inductive" means that natlist, bintree and expr are the smallest of all sets that satisfy the above typing constraints. Specifically,

*Parallel authorship, equal contribution. Corresponding author: Qinxiang Cao.

E. Pimentel and E. Tassi (Eds.): Logical Frameworks and Meta-Languages: Theory and Practice (LFMTP 2021)

EPTCS 337, 2021, pp. 55-70 doi 10.4204/EPTCS.337.5 (c) Qinxiang Cao \& Xiwei Wu

This work is licensed under the Creative Commons Attribution License. 
natlist is defined as the smallest set containing "Nil" and closed by the "Cons" constructor, which allows us to define a recursive function length on natlist so that length $\mathrm{Nil}=0$, length (Cons $\mathrm{x} 1$ ) $=1+$ length 1 , and to prove related properties by induction on the structure of natlist.

Countability is a basic property of sets. To state that a set is countable means that it has the same cardinality as some subset of the set of natural numbers. For example, in the Henkin style proof of FOL completeness [8], one important step is to construct a maximal consistent set $\Psi$ by expanding a consistent set $\Phi$ of propositions:

$$
\begin{array}{rll}
\Psi_{0} & :=\Phi & \\
\Psi_{n+1} & :=\Psi_{n} \cup\left\{\phi_{n}\right\} & \left(\text { if } \Psi_{n} \cup \phi_{n} \text { is consistent }\right) \\
\Psi_{n+1} & :=\Psi_{n} & \left(\text { if } \Psi_{n} \cup \phi_{n} \text { is inconsistent }\right) \\
\Psi & :=\bigcup_{n} \Psi_{n} & \text { where } \phi_{0}, \phi_{1}, \ldots \text { are all FOL propositions. }
\end{array}
$$

In that proof, it is critical that the set of all FOL propositions is countable. The countability property allows us to enumerate all propositions as $\phi_{0}, \phi_{1}, \ldots$ As another example, we can show the countability of computable functions by proving the countability of untyped lambda expressions. Since the set of all functions from natural numbers to natural numbers is uncountable, there must exist at least one uncomputable function. Like FO-propositions and lambda expressions, many sets can be formalized as inductive types, and we focus on the countability of the inductive types in this paper.

Proving natlist to be countable is straightforward. (1) The only natlist of length 0 is Nil. (2) The natlists of length $(n+1)$ should be countable if those natlists of length $n$ are countable (because the former set is isomorphic with the Cartesian product of $\mathbb{N}$ and the latter set). (3) By induction, the set of natlists of length $n$ is countable for any $n$. (4) The set of all natlists is a union of countably many countable sets, and thus is countable (because we can easily construct an bijection from $\mathbb{N}^{2}$ to $\mathbb{N}$ : $f(x, y)=2^{x}(2 y+1)-1$ and the construction does not even need the choice axiom). Similarly, natural number labeled binary trees with size $n$ are countable for any $n$. Thus, the elements of bintree are countable. It is natural to apply the same proof idea to a more complex inductive type. We define a rank function to generalize the length function for natlist and the size function for binary trees (with some slight modification). For example, the rank function on natlist and bintree satisfies:

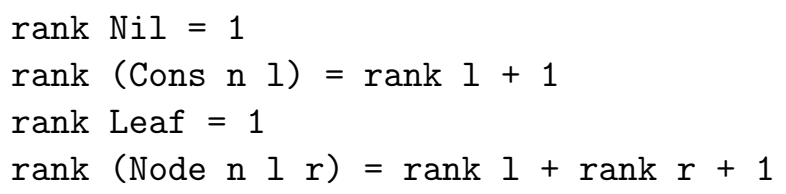

We prove that given a fixed inductive type, its elements with rank less than $n$ are countable 1 . Then, all elements of this type are also countable since the set is a union of countably many countable sets. One could write Coq tactics, which is a meta language, to describe our proof ideas above. In contrast, our target in this paper is to formally prove one single theorem in the object language for general inductive types' countability.

Handling general inductive types in Coq's object language is hard. Coq's object language, Gallina, has built-in support for recursive functions and inductive proofs, as long as they are about concrete inductive types. For general inductive types, we do not have such support, and even simple pattern match expressions are not easy to formalize. We choose to derive recursive functions and inductive proof

\footnotetext{
${ }^{1}$ In the general proof, we consider elements with rank less than $n$, not elements with rank equal to $n$
} 
principles from general recursive functions. Using concrete inductive types as an example, natlist's general recursive function is:

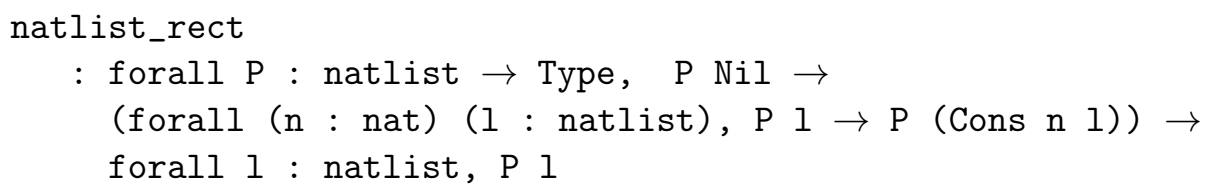

It satisfies, for any (maybe dependently typed) $P$ and $F_{0}, F_{1}$,

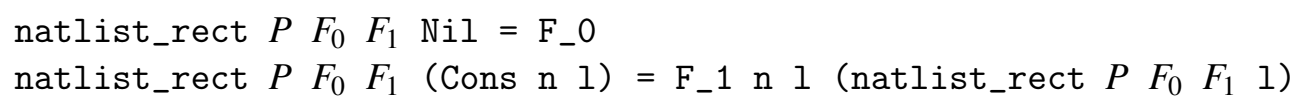

We generalize the combination of natlist_rect and the two equalities above, and develop our proofs based on them.

Theoretically, it is more difficult to do something at the object-logic level than at the meta-logic level. Any proof formalized at the object-logic level is a Coq function from its assumptions to its conclusion, according to Curry-Howard correspondence. One can always develop a corresponding meta-language function that implements the "same" functionality. In contrast, some statements are only provable in a meta-logic, but are unprovable in the object logic. Martin Hofmann and Thomas Streicher showed that the principle of uniqueness of identity proofs is not derivable in the object logic itself [12].

Practically, our proof automation, which uses object-logic proofs, is more efficient than proof generators written in a meta-language. Our tool can prove expr countable in Coq in 0.089 seconds but a tactic-based proof will take 1.928 seconds to finish the proof (see Coq development for more details). This result arises because our tool only requires Coq to typecheck one theorem with its arguments, but proofs, either proof scripts or proof terms, generated by a meta-language generator require Coq to typecheck every single proof step.

In this paper, we formalize the general proof of countability theorem mentioned above, using Coq, and automate our proof to avoid repeating the long proof process. There are several ways in which this goal may be achieved. One is to use external tools to generate the operations and proofs of corresponding lemmas. For example, DBGen [17] generates single-variable substitution operations, and Autosubst2 [20] can generate substitution-related definitions and Coq proof terms. Another approach is to use the internal facility of theorem provers, which is written in a built-in meta language, to generate proof terms or proof scripts. Brian Huffman and Alexander Krauss (old datatype), and Jasmin Blanchette (BNF datatype) have developed tactics, which is a meta language, to prove datatypes countable [21] in Isabelle/HOL.

In comparison, an object logic proof of "for any possible $T, P(T)$ holds" is one singleton proof term of type $\forall T, P(T)$. A meta-logic proof is a meta-level program (with probably more expressiveness power) which takes $T$ as its input and outputs a proof term of $P(T)$, which could be huge. Intuitively, the former one directly states that $\forall T, P(T)$ is true, while the latter one is an oracle which can step-by-step explain why $P(T)$ holds for a concrete $T$. Arthur Azevedo de Amorim's implementation [2] is the only objectlevel proof of countability before our paper. He used indices to number constructors of an inductive type. In other words, he formalized the syntax of inductive types and deeply embedded the syntax (in some sense, the meta language) in Coq's object language. As a consequence, his proof involves complicated reasoning about indices' equivalence, type's equivalence and dependent type issues-if two Coq types $T_{1}=T_{2}, T_{1}$ 's elements are not automatically recognized as $T_{2}$ 's elements by Coq's type checker. Our formalization shows that such proof-reflection technique is not needed for a general countability proof.

\footnotetext{
${ }^{2}$ Processor: $2.3 \mathrm{GHz}$ 8-Core Intel Core i9; Memory: 16 GB $2400 \mathrm{MHz}$ DDR4.
} 
Contributions. Our main contributions are a Coq formalized general countability proof for first order inductive types, and an automatic tactic for proving inductive types countable. We do not need any external tool to generate definitions, proof terms, or proof scripts, and our proof itself does not involve complicated dependently typed reasoning about type equalities. We also developed Coq libraries for countability and for a syntax of inductive definitions, which have values of their own. All of our proofs are formalized axiom-free, and our proof of countability theorem can be used in the completeness proof of separation logics, a Coq formalization for an early paper [6].

Outline. In Section 2, we will clarify our Coq definition of countability and our formalization of the syntax of first order inductive type definitions. In Section 3, we present our general countability theorem and our proof. In Section 4, we introduce our automatic tactic for proving concrete inductive types countable. We discuss related works in Section 5 and conclude in Section 6 ,

\section{Preliminaries}

In this section, we present our formal definition of countable (Section 2.1), the syntax of first order inductive types (Section 2.2), and general recursive functions (Section 2.3). We will also list their important properties, that we prove in our Coq library.

\subsection{Countable}

We define the type $\mathrm{T}$ to be countable if and only if there exists an injection from $\mathrm{T}$ to natural numbers, which means $\mathrm{T}$ is either finite or countably infinite. Here, an injection is a relation that keeps the injective property and functional property. This Countable is the definition used in our final theorem, but we use an auxiliary definition, SetoidCountable, in our proof. For the countability proof of inductive type $T$, we need to prove that $\{x: T \mid \operatorname{rank}(x)<n\}$ is countable for any $n$. In Coq, an element in $\{x: T \mid \operatorname{rank}(x)<n\}$ is a dependently typed tuple $(x, p)$, where $x \in T$ and $p$ is a proof of $\operatorname{rank}(x)<n$. Two such dependently typed tuples $\left(x_{1}, p_{1}\right)$ and $\left(x_{2}, p_{2}\right)$ are equal if $x_{1}=x_{2}$, and $p_{1}$ and $p_{2}$ are identical proof terms. Proving $\{x: T \mid \operatorname{rank}(x)<n\}$ Countable requires us to show whether two proofs, $p_{1}$ and $p_{2}$, of $\operatorname{rank}(x)<n$ are identical. Using SetoidCountable avoids that kind of reasoning about proof terms, and avoids using the "proof-irrelevance" axiom in some sense 3 . The definition of $\{x: T \mid \operatorname{rank}(x)<n\}$ being SetoidCountable is straightforward: there exists a function $f$ from $\{x: T \mid \operatorname{rank}(x)<n\}$ to natural numbers, so that if $f\left(x_{1}, p_{1}\right)=f\left(x_{2}, p_{2}\right)$ then $x 1=x 2$.

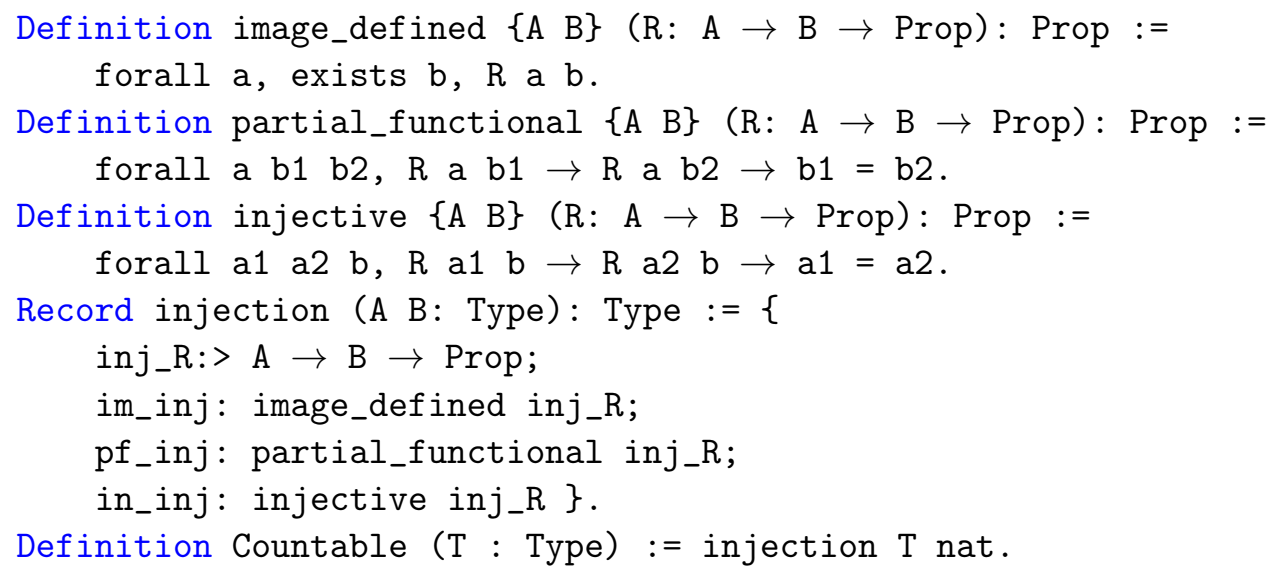

${ }^{3}$ Axiom proof_irrelevance : forall (P:Prop) (p1 p2:P), p1 = p2. 


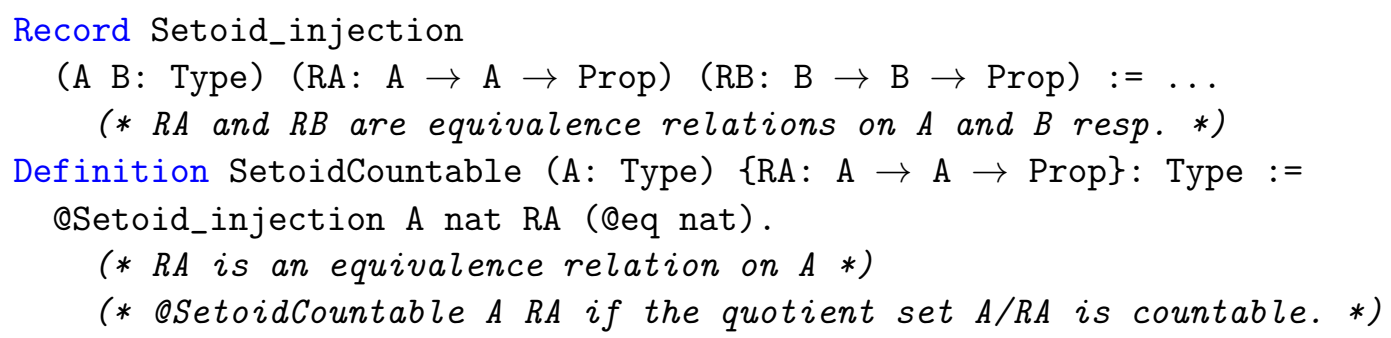

In our countability library, we prove that products of two countable types and unions of countably many countable types are countable. We prove that the composition of two injections is still an injection (see injective_compose below), and if $f \circ g$ is an injection then $f$ is an injection (see injective_compose_rev below). We also prove their setoid versions, but omit them here. We define "bijection" and prove some elementary properties about bijection and injection. For connections between SetoidCountable and Countable, we prove that any Setoid_injection on Coq's builtin equality is an injection, and thus any SetoidCountable type w.r.t. Coq's builtin equality is also Countable.

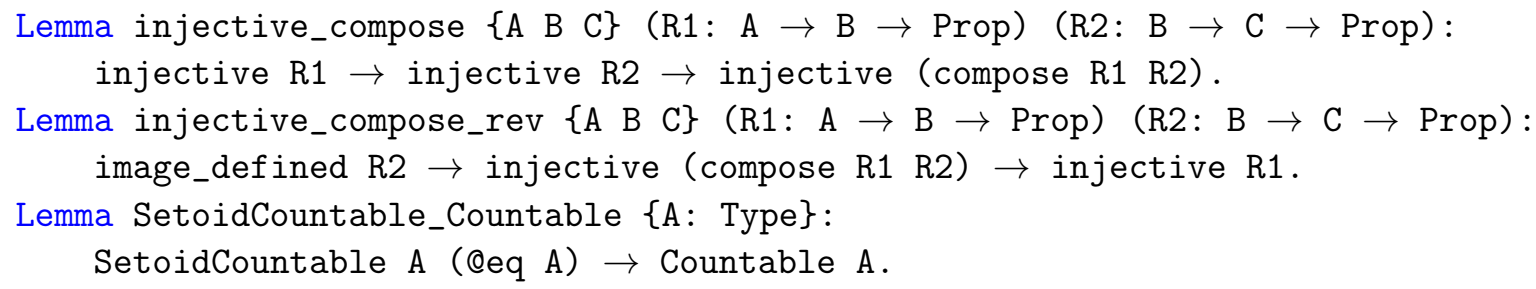

Here, we use a relation rather than a function to provide the definition of Countable, for better usability and extensibility. For example, if we have a bijection from $A$ to $B$ and we have Countable B, we want to show that $A$ is also countable. We can do it easily by relation, but we cannot do it under the definition of function, because of the problem of computability.

\subsection{Syntax of inductive definition}

In our formalization, we only consider first order inductive definitions. Not all inductive types have only countably many elements. Thus we exclude definitions like the following:

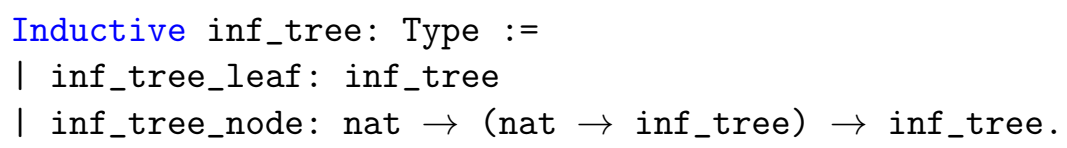

However, we try to focus on techniques of building dependently type functions from inductive definitions to nontrivial proof terms in this work. Thus we choose to exclude mutually inductive definitions and nested inductive definitions from our formalization, although we believe that we can extend our work in the future to handle these cases.

Formally, a first-order inductive type $T$ is defined by a list of constructors, each of which is a firstorder function with result type $T$. The argument types of constructors should be constant base type 4 or $T$ itself. So we formalize the syntax of an inductive definition $T$ as a list of dependently typed pairs of typing rules and constructors: list (sigT ( $f u n$ arg $\Rightarrow$ constr_type arg T)), we will call it Constrs_type later in this paper. We usually call the "typing rule" part arg, with a type list (option Type), and call a constructor constr, whose type depends on arg and is calculated

\footnotetext{
${ }^{4}$ Here, constant base type means other types which are countable.
} 
by constr_type. For example, the definition of natlist has two branches. The type of Cons is: nat $\rightarrow$ natlist $\rightarrow$ natlist. It has two arguments, one of which is of type nat and the other is the inductive type natlist itself. Thus, this typing rule can be formalized as: [Some nat; None] (Some for a based type and None for the inductive type itself) and

constr_type [Some nat; None] natlist $=$ nat $\rightarrow$ natlist $\rightarrow$ natlist

exactly describes the type of constructor Cons. Since the definition of natlist has two branches, this definition can be described by: [ _ [ [Some nat; None $]$, Cons $]_{-}$; $\left.[\text {[], Nil }]_{-}\right]_{\text {. }}$

\subsection{General recursion}

For an inductive type $\mathrm{T}$, Coq generates $\mathrm{T}_{-}$rect, $\mathrm{T}_{-}$ind, $\mathrm{T}_{-} \mathrm{rec}$ and $\mathrm{T}_{-}$sind, which respectively correspond to elimination principles on Type, Prop, Set and SProp[5]. For our countability proof, T_rect is enough 5 . We define rect_type to compute the type of $\mathrm{T}_{-}$rect (we call it rect later in this paper) from an inductive definition. Similar to the definition of Constrs_type, we use rect_clause_type to compute each branch. Here we show the definitions of rect_type :

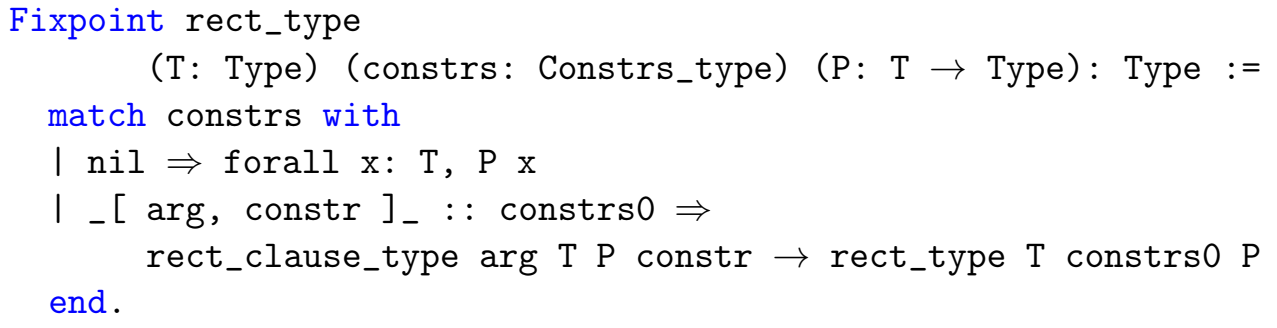

For example, natlist_rect (which is generated by Coq) has type

forall P, rect_type natlist [ _ [ [Some nat; None $]$, Cons $\left.]_{-} ;{ }_{-}[[], \mathrm{Nil}]_{-}\right] \mathrm{P}$

Knowledge of the type of general recursive function alone is not sufficient for building proofs. It is important that the computation result of a recursive function coincides with the definitions in its corresponding branch. For example, as mentioned in Section 1, natlist_rect satisfies:

natlist_rect $P \quad F_{0} \quad F_{1}$ Nil $=F_{-} 0$

natlist_rect $P F_{0} F_{1}$ (Cons $\mathrm{n} 1$ ) $=\mathrm{F}_{-} 1 \mathrm{n} 1$ (natlist_rect $P F_{0} F_{1}$ l)

For general inductive types, we need to consider all possible ways of filling rect's arguments. We introduce apply_rect so that apply_rect T P constrs para (rect P) x fills rect's argument (like $F_{0}$ and $F_{1}$ above) in a parameterized way defined by para and calculates the result on $x: T$. Based on that, we define rect_correct:

$$
\begin{aligned}
& \text { rect_correct (T: Type) (constrs: Constrs_type) } \\
& \text { (rect: forall P, rect_type T constrs P): Prop }
\end{aligned}
$$

to be the following property: for any para and $\mathrm{x}$, apply_rect $\mathrm{T} P$ constrs para (rect $\mathrm{P}$ ) $\mathrm{x}$ equals to the recursive branch defined by para and $\mathrm{x}$ (and the recursive function apply_rect $\mathrm{T} P$ constrs para rect itself). Detailed definitions of apply_rect and rect_correct involve complicated dependent type issue, and we defer them to Section 3 .

\footnotetext{
${ }^{5}$ The constant $T_{-}$ind is always generated, whereas $T_{-} r e c$ and $T_{-} r e c t$ may be impossible to derive, for example, when the sort is Prop. However, we only focus on the inductive type so no problems are encountered.
} 
In summary, our proof about inductive type's countability depends on and only depends on the following arguments and hypothesis:

- the Coq type T: Type;

- the inductive definition constrs: Constrs_type;

- the general recursive function rect: forall P, rect_type T constrs P;

- the characteristic equations rect_correctness: rect_correct constrs rect.

We develop three automatic tactics gen_constrs, gen_rect, apply_rect_correctness_gen to get constrs, rect and rect_correctness above from T.

- In order to get rect, we build a virtual induction proof on $\mathrm{T}$ and analyze that proof term. For example, one can prove "forall 1: natlist, Type" by the following tactic:

intro 1 ; induction 1 ; exact bool.

This tactic will generate the following proof term:

fun 1 : natlist $\Rightarrow$ natlist_rect (fun _ $\Rightarrow$ Type) bool (fun _ _ $\Rightarrow$ bool) 1

Then our tactic analyzes this proof term to get natlist_rect.

- In order to get constrs, we get rect first and analyze the syntax of its type. For example,

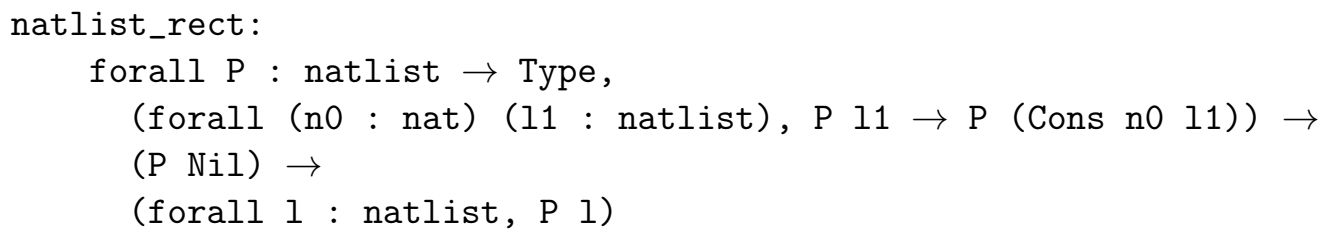

From its assumptions, our tactic can generate

[ _ [ [Some nat; None $],$ Cons $]_{-} ;$_ $\left.\left._{[}[], \mathrm{Nil}\right]_{-}\right]$.

- In order to get rect_correctness, we only need to unfold the definitions of rect_correct and apply_rect, and prove the conclusion by reflexivity.

\section{The countability theorem}

As mentioned in Section 1, the main proof idea is to define a rank function from inductive type $\mathrm{T}$ to nat, and prove that $T_{n} \triangleq\{x: T \mid \operatorname{rank}(x)<n\}$ is countable for any $n$. This conclusion can be proved by induction on $n$. Its induction step is to construct an injection from $T_{n+1}$ to the union of different products of $T_{n}$, and the latter one is countable since $T_{n}$ is countable by the induction hypothesis. Using natlist and bintree as examples, we can construct injections:

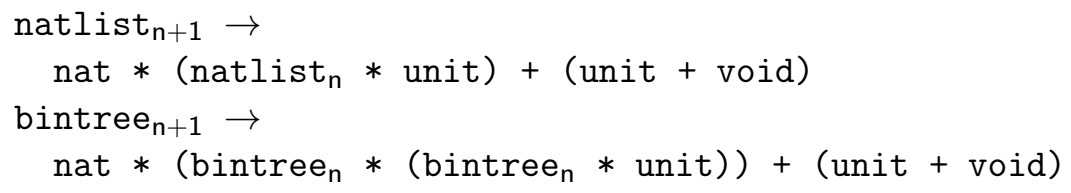

\footnotetext{
${ }^{6}$ Here we choose not to present the correct rank function, for reasons of conciseness. The real general rank function takes more arguments to be specialized on natlist and bintree; see Section 3.1 Also, redundant "* unit" and "+ void" are introduced by a uniform recursive definition for convenience.
} 
Using natlist as an example, this construction of injection takes three steps:

- Defining a function from natlist to nat $*$ (natlist $*$ unit $)+($ unit + void):

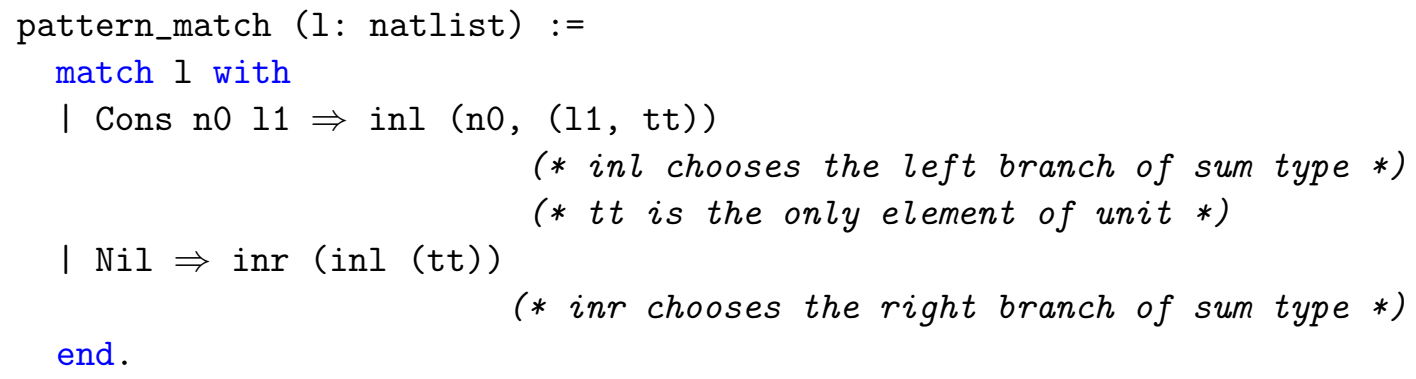

- Well-definedness of pattern_match:

We thus prove that if rank $l<S n$ and pattern_match $l=\operatorname{inl}(n 0,(11, t t))$, then rank $11<\mathrm{n}$. Thus, we can define a dependently typed function of the type below based on pattern_match.

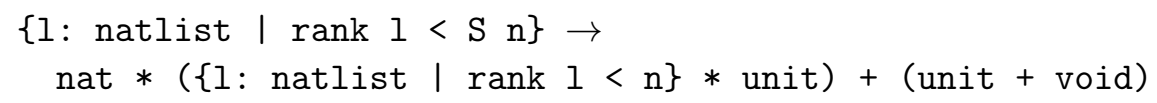

In other words, we define

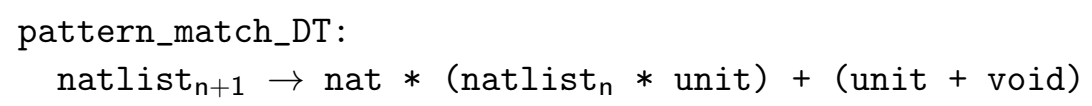

- Injective property:

We prove that the pattern_match function we defined is an injection.

In Section 3.1, we introduce our general definition of rank and pattern_match. In Section 3.2 and 3.3. we establish the injective property above. Specifically, we first prove that pattern_match itself is an injection (see Section 3.2) and use that conclusion to prove our final dependently typed version injective (see Section 3.3). Finally, we summarize our main theorem in Section 3.4.

\subsection{Definitions}

We first define the function that calculates the union of different products named normtype. As shown in the beginning of section 3, we want to project $T_{n+1}$ into the union of different products of $T_{n}$. Also, the non-dependent type version pattern_match is a function from $T$ to the union of different products of $T$. Thus, our definition of normtype is polymorphic. For example,

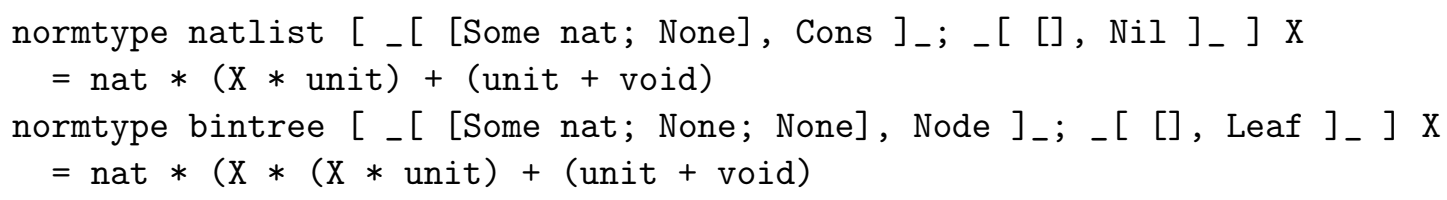

In our definition of normtype, we analyze the inductive definition constrs (which means [ _ [ [Some nat; None $]$, cons $]_{-}$; $\left._{-}[[] \text {, nil }]_{-}\right]$for natlist), use product types to represent each branch, and use sum types to connect them. For each branch, we use $A$ when we meet Some A and use $X$ when we meet None. We can therefore generate all normtype types with the same syntax tree as $T$ like $T_{n+1}$. 
As mentioned in Section 2, we need to define all recursive function (e.g. rank) and pattern match expressions (e.g. pattern_match) based on the general recursive function. Specifically, suppose rect is the general recursive function of type $T$ with inductive definition constrs, we define rank and pattern_match by filling rect's arguments through apply_rect.

When defining rank, each argument of rect is to add recursive calls' results together. For example, size (see Section 1) is the rank function of bintree. We can define it by filling bintree's arguments:

$$
\text { size } t=\text { bintree_rect _ (fun n0 t1 r1 t2 r2 } r \text { r1 }+r 2+1)
$$

Here, in the recursive branch for constructor "Node", $\mathrm{n} 0$ is the label, t1 and t2 are the left and right subtrees respectively, and $r 1$ and $r 2$ are the results of recursive calls: size $t 1$ and size $t 2$; and in the recursive branch of constructor "Leaf", the return value is a constant 1. In general, we can define these arguments of rect based on the syntax of inductive definitions. Using the example above, the typing information of "Node" is described by [Some nat; None; None] since Node has type:

$$
\text { nat } \rightarrow \text { bintree } \rightarrow \text { bintree } \rightarrow \text { bintree }
$$

The first element Some nat corresponds to $\mathrm{n} 0$ above; the second element None corresponds to $\mathrm{t} 1$ and $r 1$ above; and the element None corresponds to t2 and $r 2$.

Defining pattern_match is more complicated. Here is how we define the pattern_match function for natlist (see the beginning of Section 3) by filling rect's arguments.

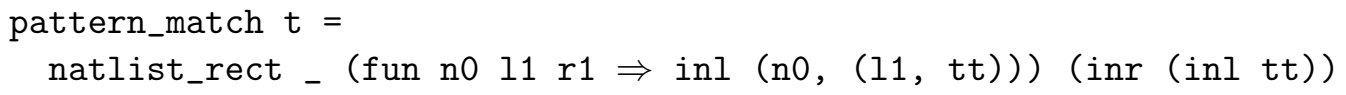

Here, we put inl in the Cons branch since it is the first branch, and we put inr $\circ$ inl in the Nil branch since it is the second branch. That means we cannot define these two arguments of natlist_rect based only on Cons's and Nil's typing information. Specifically, if constrs can be decomposed into: constrs1 ++ _ [arg2, constr2] _ : constrs3, then rect's arguments for the arg2-constr2branch also depend on the length of constrs1 and constrs3. For this reason, the definition of apply_rect must allow para (the parameterized way of filling rect's arguments) to take constrs 1 and constrs 3 as its arguments.

In our real definition, apply_rect's type is:

$$
\text { apply_rect } \mathrm{T} \text { constrs } \mathrm{P} \text { para rect_P : forall ( } \mathrm{t}: \mathrm{T}), \mathrm{P} \text { constrs } \mathrm{t} \text {. }
$$

where (we omit para's type first and introduce it later)

$$
\begin{aligned}
& \mathrm{P}: \text { Constrs_type } \rightarrow \mathrm{T} \rightarrow \text { Type; } \\
& \text { rect_P: rect_type } \mathrm{T} \text { constrs ( } \mathrm{P} \text { constrs) }
\end{aligned}
$$

Here P defines the dependently typed result type and rect_P is a specialized rect for computing results of "type" P. The most important parameter of apply_rect is para. Its type is:

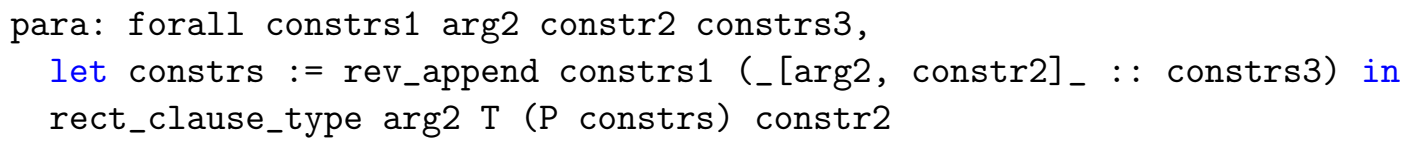

That is: if constrs, all branches of inductive definitions, can be decomposed into (rev constrs1), _ [arg2, constr2] _, and constrs3, then para computes rect_P's argument for the branch of arg2 and constr2. Here, the function rev_append is the auxiliary function for defining a tail-recursive list reverse provided by Coq's standard library: 


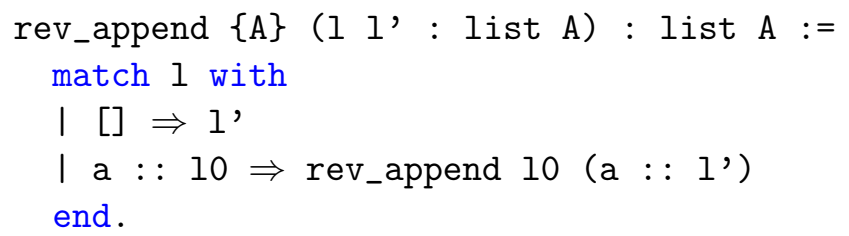

It ensures that for any 1 and $l^{\prime}$, rev_append $1 l^{\prime}=\operatorname{rev} 1++1$. It is critical for us to use

rev_append constrs1 (_[arg2, constr2]_ : : constrs3)

instead of

constrs1 ++ _ [arg2, constr2]_ : constrs3

because it is much more convenient for building dependently typed inductive proofs and dependently typed recursive functions. When we apply an inductive proof on the structure of constrs, we can easily transform

$$
\begin{aligned}
& \text { rev_append constrs1 (_[arg2, constr2]_ : : constrs3) } \\
& \text { to rev_append (_[arg2, constr2]_ : : constrs1) constrs3 }
\end{aligned}
$$

because they are $\beta \eta \imath$-reduction to each other, and so can pass Coq's unification checking. But it is not the case for

$$
\begin{aligned}
& \text { constrs1 ++ (_[arg2, constr2 }]_{-}::_{\text {c constrs } 3)} \\
& \text { and (constrs1 ++ } \left.\left[\ldots[\arg 2, \text { constr2 }]_{-}\right]\right)++ \text {constrs3 }
\end{aligned}
$$

which are equal, and can be proved equal, but they cannot pass Coq's unification checking.

In the end, we define paras for rank and pattern_match, and define these two functions for generic inductive types based on corresponding paras and apply_rect.

\subsection{Injective property: the simple typed version}

We have defined pattern_match, a function from $T$ to normtype $T$ constrs $T$ (which is a union type of different product types). We prove it to be an injection in this subsection. Later, we will use this simplified conclusion to establish our ultimate goal: a Setoid_injection from $\mathrm{T}_{n+1}$ to normtype $\mathrm{T}$ constrs $\mathrm{T}_{n}$.

To prove pattern_match to be injective (formalized as PM_inj below), we need to perform case analysis over both $a$ and $b$.

Definition PM_inj $\mathrm{T}$ constrs rect: Prop :=
forall a b: T, pattern_match $\mathrm{a}=$ pattern_match $\mathrm{b} \rightarrow \mathrm{a}=\mathrm{b}$.

Again, case analysis proofs is nontrivial since we are now reasoning about a generic inductive type, not a concrete one. We use a specialized rect to solve the problem:

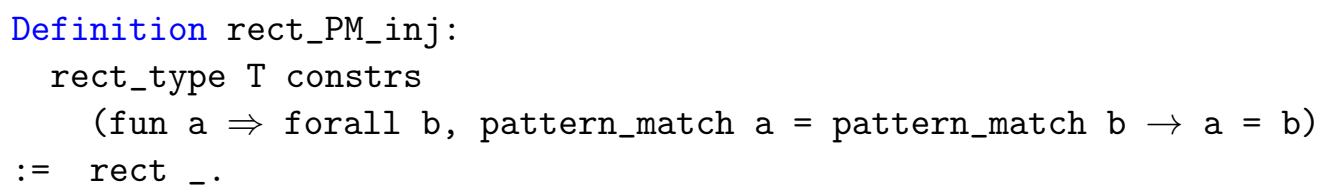

Here, rect_PM_inj is a proof of a big implication proposition, whose conclusion is exactly PM_inj and whose assumptions are those case analysis branches. Thus, the proof of the injective property can be built by filling all those arguments of rect_PM_inj. Specifically, we decompose constrs into a form of rev_append constrs1 constrs2 (at first constrs1 $=[]$ and constrs 2 = constrs) and fill those 
arguments by an induction over constrs 2 . We successfully avoid dependently typed type-casting since we use rev_append in this proof and in apply_rect's types. We omit proof details here.

We now finish the definition of a "simple" typed function, pattern_match, and the proof of its injective property. During the proof process, we encounter some problems regarding dependent types and solve them with the help of rev_append. To construct an injection from $T_{n+1}$ (defined as $\left\{\begin{array}{l}\mathrm{x}: \mathrm{T} \\ \text { I }\end{array}\right.$ rank $\mathrm{x}<\mathrm{S} \mathrm{n}\}$ ) to normtype $\mathrm{T}$ constrs $\mathrm{T}_{n}$, we need to prove linear arithmetic properties about rank. We could prove that dependently typed injective property in a similar way, but the dependent types with irrelevant proofs will trouble us much more. So we choose a different proof strategy.

\subsection{Injective property: the dependent type version}

Instead of developing a similar (but more complicated) proof of the injective property for the dependently typed version of pattern_match, we choose to use our injective proof above to build our dependently typed injective proof. In order to prove pattern_match_DT (the dependently typed version of pattern_match, a mapping from $\mathrm{T}_{n+1}$ to normtype $\mathrm{T}$ constrs $\mathrm{T}_{n}$ ) to be injective, it is sufficient to show (Fig. 11illustrates this proof strategy):

- pattern_match $\circ$ proj1_sig = (normtype_map proj1_sig) $\circ$ pattern_match_DT;

- pattern_match is injective;

- proj1_sig is injective.

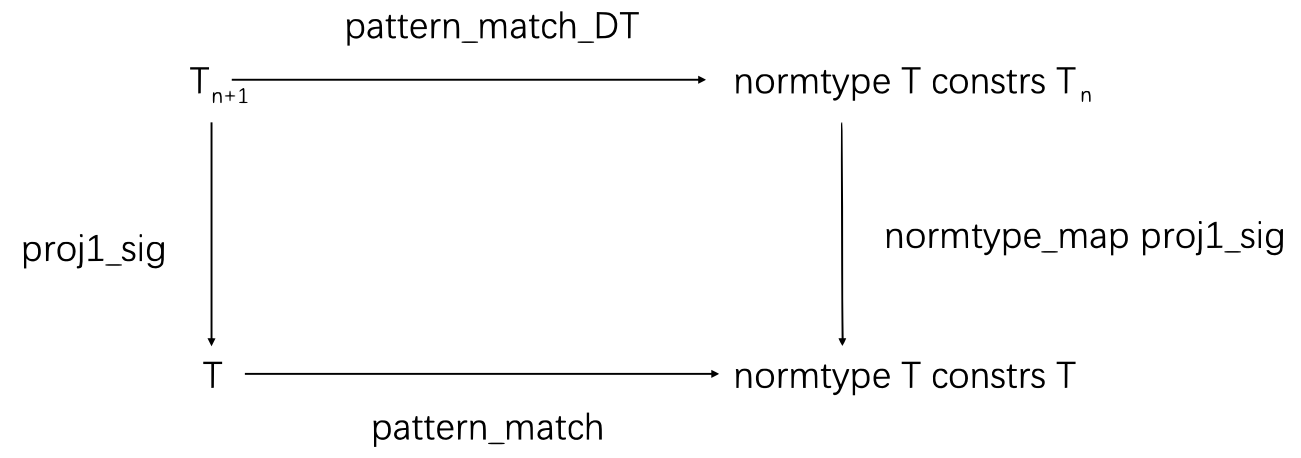

Figure 1: Proof strategy

Here, proj1_sig (defined by Coq standard library) removes the proof part of Coq's sigma types:

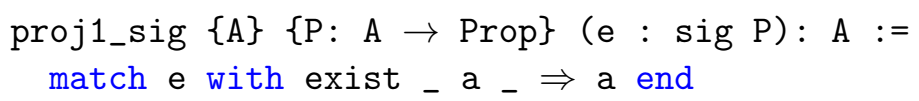

and we define normtype_map to apply proj1_sig on each part of normtype $T$ constrs $T_{n}$.

The reasoning behind this proof strategy is straightforward. By the second and the third condition (pattern_match and proj1_sig are injective), we know that pattern_match $\circ$ proj1_sig is injective using theorem injective_compose (see Section 2.1). Thus, (normtype_map proj1_sig) o pattern_match_DT is also injective according to the first condition (the diagram in Fig. 11 commutes). In the end, pattern_match_DT must be an injection due to theorem injective_compose_rev (see Section 2.11.

We proved pattern_match to be injective in Section 3.3 and proj1_sig is obviously an injection; thus we only need to prove pattern_match $\circ$ proj1_sig = (normtype_map proj1_sig) 0 pattern_match_DT, based on our definition of pattern_match_DT and normtype_map. 
In our Coq formalization, the real type of pattern_match_DT is:

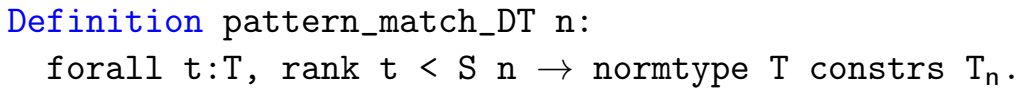

given type $T$, its inductive definition constrs and its general recursive function rect and rect_correctness. Using natlist as an example,

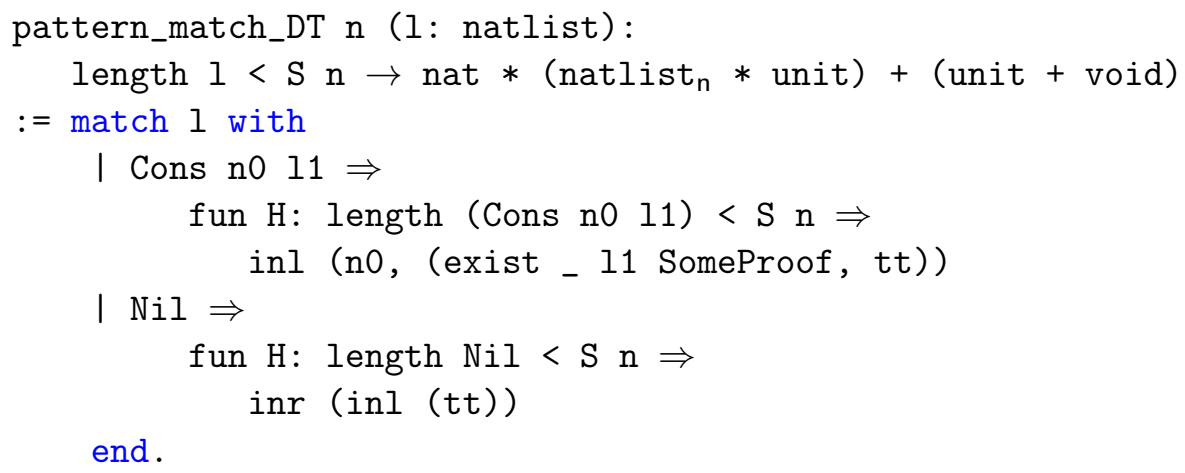

The function above may be hard to read. It is equivalent to the following one, which is written in a less dependently typed way.

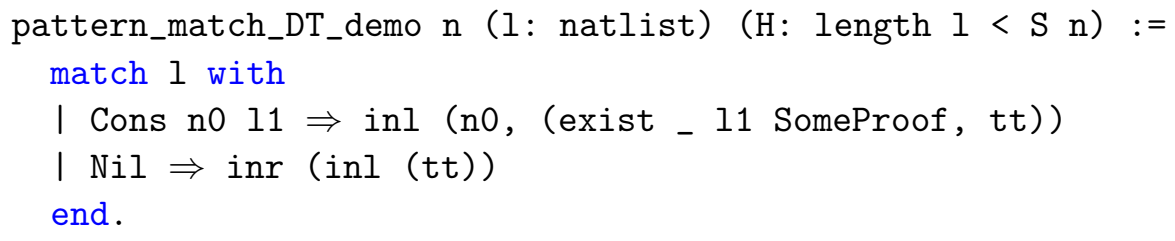

In their Cons branches, we need to provide a proof of length $11<\mathrm{n}$ at the place of SomeProof given $\mathrm{H}$ : length $1<\mathrm{S}$.

In order to define such a pattern_match_DT function for a generic inductive type T, we build its definition based on apply_rect and a "para" of the following type:

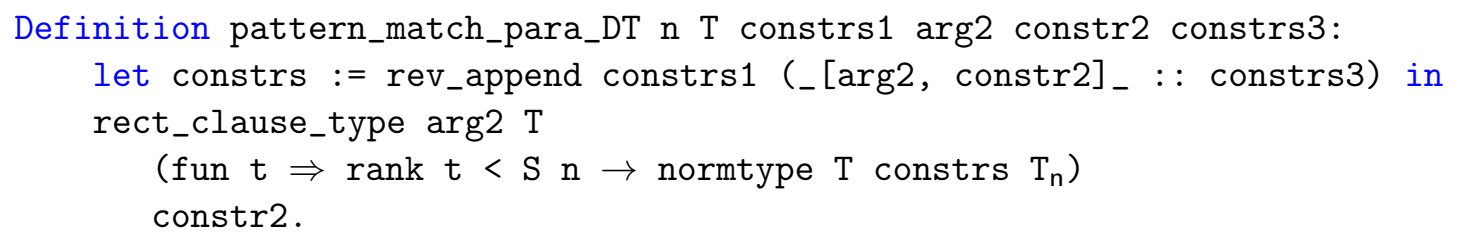

That is, we define pattern_match_DT by filling arguments of T's general recursive function rect. Given that constrs is decomposed into rev_append constrs1 (_[arg2, constr2]_ : : constrs3), this para above computes the arg2-constr2-argument of rect. Again, using the Cons argument of natlist_rect as an example, this para should have the following type:

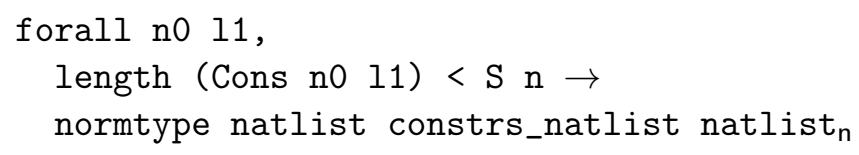

In the definition of pattern_match_para_DT and the proof of pattern_match $\circ$ proj1_sig $=$ (normtype_map proj1_sig) o pattern_match_DT, we repeatedly use rect_correctness to reason about rank. For the sake of space, we omit details here. 


\subsection{Main theorem}

We have constructed the injection from $\mathrm{T}_{n+1}$ to normtype $\mathrm{T}$ constrs $\mathrm{T}_{n}$ in Coq. The definition of apply_rect helps us define most of the functions we need, and most of the intermediate lemmas can be reduced to properties of apply_rect and the corresponding para, which greatly simplifies our proof. We use rev_append to avoid type casting.

In summary, we use SetoidCountable as an auxiliary definition to prove the countability theorem. Specifically, we prove SetoidCountable $\mathrm{T}_{n}$ by induction over $n$ and by the injection we just constructed. In the end, our main theorem is:

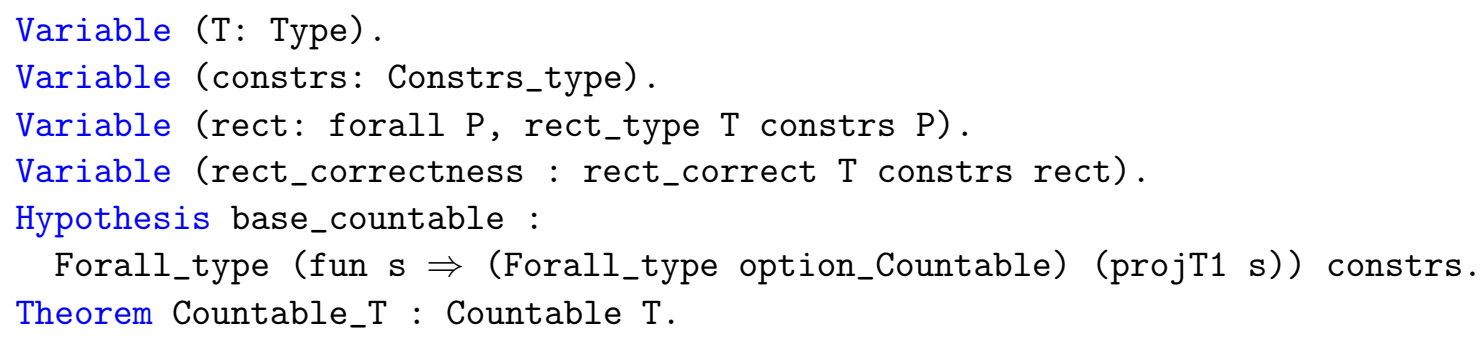

where the function Forall_type (provided by Coq's standard library) defines the universal predicates over lists

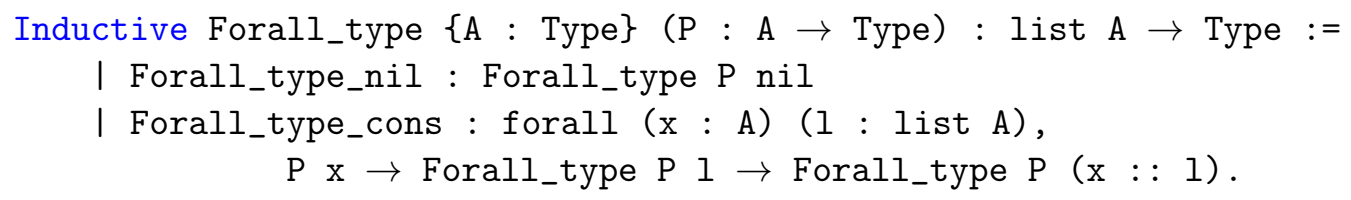

and the hypothesis base_countable says that all base types used in the inductive definition are countable. For example, this theorem proves that natlist is countable, as long as nat is countable.

\section{Automatic proof systems}

To make our result more practical and accessible, we developed Coq tactics to automatically prove inductive types countable. We define three tactics gen_constrs, gen_rect, apply_rect_correctness_gen to get constrs, rect and rect_correctness from T (previously mentioned in Section 2, see Coq development for more details). According to the list of conditions and hypothesis, we need in proof (as shown above in Section 3.4), the last thing that needs to be done is to prove base_countable, which means that all base types used in the definition of $\mathrm{T}$ are countable. This is done using a library of proved countability results and Coq assumptions. Here are two typical applications of our tactic Countable_solver:

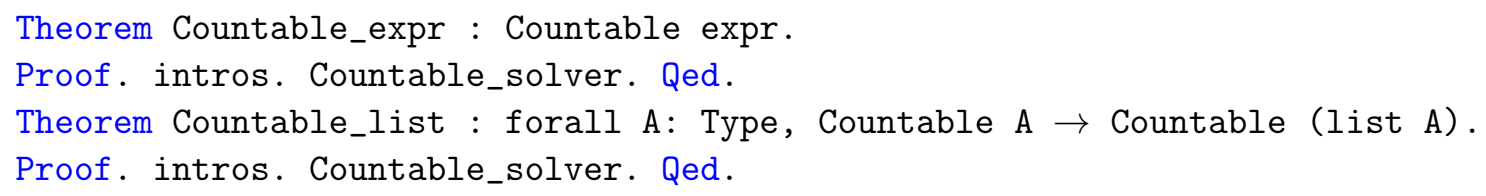




\section{Related work}

We discuss three related studies in proving relevant properties for inductive types in Coq and one in Isabelle/HOL. What sets our formalization apart from this work is our proof at Coq's object logic level; only Deriving [2] develops object logic proofs like us. As a result, our proofs and automation instructions do not need other additional axioms, libraries, or tools, and can run with high efficiency.

- Theory Countable[1] : These researchers also used injection to represent the Countable relation. Their main idea was to construct an injection from data types to old data types which are countable. They could automatically prove the countability of data types which had nested and mutual recursion, and used other data types. However, the process and automatic tactics were formalized in a meta language.

- Autosubst[19] \& Autosubst2[20]: Autosubst can automatically generate the substitution operations for a custom inductive type of terms, and prove the corresponding substitution lemmas. The library gives the enumerability of De Brujin substitution algebra[18]. Autosubst offers tactics that implement the normalization and decision procedure. They believe that it is hard to maintain or extend Ltac code, so that they proposed a new implementation of Autosubst which comes in the form of a code generator to generate Coq proof terms, and at the same time extends Autosubst's input language to mutual inductive sorts with multiple sorts of variables. Autosubst and Autosubst2 develop formalized proofs as tactics and external proof term generators, respectively, which can be treated as meta-level mappings from the syntax of inductive definitions to countability proof terms. In comparison, our proof is a Coq object-level mapping from inductive definitions to countability, but we do not support mutually inductive types at present.

- Undecidability[9] : Forster et. al. formalized the computational undecidability of the validity, satisfiability, and provability of first-order formulas following a synthetic approach based on the computation native to Coq's constructive type theory. They extended the library in 2020, to present a comprehensive analysis of the computational content of completeness theorems for first-order logic, considering various semantics and deduction systems[10]. They proved first-order logic's propositions countable in their completeness proof. They also formalized the syntax of inductive definitions 8 , as we did, but they do not provide a general theorem of countability.

- Deriving [2] : Deriving proved inductive types countable in Coq. Deriving uses countType in the MathComp library, which provides a different definition of injection and Countable. Although this alternative definition requires injections to be Coq-computable functions, it is not a significant drawback comparing with our definitions when applying inductive type's countability. Deriving supports the proof of countability for mutually inductive types and nested inductive types. Their proof strategy is different from ours: they built an injection from every inductive type $T$ to finitewidth trees, which they defined as a Coq type and proved countable. More significantly, they provided two formalization of inductive definitions. One is like ours:

constrs: list (sigT (fun $\arg \Rightarrow$ constr_type $\arg \mathrm{T}$ ));

the other uses indices to number the constructors. In other words, the latter formalization is a deep embedding of the meta language (the syntax of inductive types) into Coq's object language. They

\footnotetext{
${ }^{7}$ This general formalization appears in their Coq development but they do not mention that in their paper.
} 
carefully used computable functions over the deeply embedded meta language (since natural numbers' equality tests are computable, but types' equality tests are not computable) in their definitions and used the connection between these two formalizations to compute their final proof term. In their proofs, they need to reason about indices' equalities, types' equalities and relevant dependent type issues-if two Coq types $T_{1}=T_{2}, T_{1}$ 's elements are not automatically recognized as $T_{2}$ 's elements by Coq's type checker. In comparison, our work shows that inductions over constructor lists do prove the conclusion using the "rev-append trick", and we do not need number-indexing and heavy-weighted proof reflection to bypass related difficulties in dependently type proofs.

\section{Conclusions}

We proved in Coq that a first-order inductive type is countable as long as all base types used in the definition are countable. Our definitions and proofs are all axiom-free. We provide an alternative way of thinking about solving dependent types at the object level. We developed very efficient tactics which use this countability theorem to prove concrete inductive types to be countable. Our formalization and automatic tactic still have room for expansion in the future. For example, our tactics do not work when applied to mutually recursive types. We believe that it is plausible to transform the mutually recursive types into primitive recursive types [11] and enhance our tactics. Our Coq development can be found at: https://github.com/QinxiangCao/Countable_PaperSubmission

Ackownledgement This research is sponsored by National Natural Science foundation of China (NSFC) Grant No. 61902240 and Shanghai Pujiang Program 19PJ406000.

\section{References}

[1] Jasmin Blanchette Alexander Krauss, Brian Huffman: This is a library of Countable Theory in Isabelle/HOL. https://devel.isa-afp.org/browser_info/current/HOL/HOL-Library/Countable.html

[2] A. A. de Amorim (2020): Deriving Instances with Dependent Types. CoqPL 2020.

[3] Abhishek Anand \& Vincent Rahli (2014): A Generic Approach to Proofs about Substitution. In Amy P. Felty \& Brigitte Pientka, editors: Proceedings of the 2014 International Workshop on Logical Frameworks and Meta-languages: Theory and Practice, LFMTP '14, Vienna, Austria, July 17, 2014, ACM, pp. 5: 1-5: 8, doi $10.1145 / 2631172.2631177$

[4] Brian Aydemir \& Stephanie Weirich (2010): LNgen: Tool support for locally nameless representations.

[5] Bruno Barras, Samuel Boutin, Cristina Cornes, Judicaël Courant, Yann Coscoy, David Delahaye, Daniel de Rauglaudre, Jean-Christophe Filliâtre, Eduardo Giménez, Hugo Herbelin et al. (1999): The Coq proof assistant reference manual. INRIA, version 6(11).

[6] Qinxiang Cao, Santiago Cuellar \& Andrew W. Appel (2017): Bringing Order to the Separation Logic Jungle. In Bor-Yuh Evan Chang, editor: Programming Languages and Systems - 15th Asian Symposium, APLAS 2017, Suzhou, China, November 27-29, 2017, Proceedings, Lecture Notes in Computer Science 10695, Springer, pp. 190-211, doi:10.1007/978-3-319-71237-6_10.

[7] Adam Chlipala (2008): Parametric higher-order abstract syntax for mechanized semantics. In James Hook \& Peter Thiemann, editors: Proceeding of the 13th ACM SIGPLAN international conference on Functional programming, ICFP 2008, Victoria, BC, Canada, September 20-28, 2008, ACM, pp. 143-156, doi: $10.1145 / 1411204.1411226$

[8] Heinz-Dieter Ebbinghaus, Jörg Flum \& Wolfgang Thomas (1994): Mathematical logic (2. ed.). Undergraduate texts in mathematics, Springer, doi:10.1007/978-1-4757-2355-7. 
[9] Yannick Forster, Dominik Kirst \& Gert Smolka (2019): On synthetic undecidability in coq, with an application to the entscheidungsproblem. In Assia Mahboubi \& Magnus O. Myreen, editors: Proceedings of the 8th ACM SIGPLAN International Conference on Certified Programs and Proofs, CPP 2019, Cascais, Portugal, January 14-15, 2019, ACM, pp. 38-51, doi $10.1145 / 3293880.3294091$.

[10] Yannick Forster, Dominik Kirst \& Dominik Wehr (2020): Completeness Theorems for First-Order Logic Analysed in Constructive Type Theory. In Sergei N. Artëmov \& Anil Nerode, editors: Logical Foundations of Computer Science - International Symposium, LFCS 2020, Deerfield Beach, FL, USA, January 4-7, 2020, Proceedings, Lecture Notes in Computer Science 11972, Springer, pp. 47-74, doi:10.1007/978-3-030-36755-8_4

[11] Peter Freyd (1990): Recursive types reduced to inductive types. In: [1990] Proceedings. Fifth Annual IEEE Symposium on Logic in Computer Science, pp. 498-507, doi-10.1109/LICS.1990.113772.

[12] M. Hofmann \& T. Streicher (1994): The groupoid model refutes uniqueness of identity proofs. In: Proceedings Ninth Annual IEEE Symposium on Logic in Computer Science, pp. 208-212, doi:10.1109/LICS.1994.316071.

[13] Gyesik Lee, Bruno C. d. S. Oliveira, Sungkeun Cho \& Kwangkeun Yi (2012): GMeta: A Generic Formal Metatheory Framework for First-Order Representations. In Helmut Seidl, editor: Programming Languages and Systems - 21st European Symposium on Programming, ESOP 2012, Held as Part of the European Joint Conferences on Theory and Practice of Software, ETAPS 2012, Tallinn, Estonia, March 24 - April 1, 2012. Proceedings, Lecture Notes in Computer Science 7211, Springer, pp. 436-455, doi:10.1007/978-3-642-28869-2_22

[14] Leonardo Mendonça de Moura, Soonho Kong, Jeremy Avigad, Floris van Doorn \& Jakob von Raumer (2015): The Lean Theorem Prover (System Description). In Amy P. Felty \& Aart Middeldorp, editors: Automated Deduction - CADE-25 - 25th International Conference on Automated Deduction, Berlin, Germany, August 1-7, 2015, Proceedings, Lecture Notes in Computer Science 9195, Springer, pp. 378-388, doi:10.1007/978-3-319-21401-6_26

[15] Rob Nederpelt \& Herman Geuvers (2014): Type theory and formal proof: an introduction. Cambridge University Press, doi $10.1017 / \mathrm{CBO} 9781139567725$,

[16] Christine Paulin-Mohring (2015): Introduction to the Calculus of Inductive Constructions. In Bruno Woltzenlogel Paleo \& David Delahaye, editors: All about Proofs, Proofs for All, Studies in Logic (Mathematical logic and foundations) 55, College Publications. Available at https://hal .inria.fr/hal-01094195.

[17] Emmanuel Polonowski (2013): Automatically Generated Infrastructure for De Bruijn Syntaxes. In Sandrine Blazy, Christine Paulin-Mohring \& David Pichardie, editors: Interactive Theorem Proving - 4th International Conference, ITP 2013, Rennes, France, July 22-26, 2013. Proceedings, Lecture Notes in Computer Science 7998, Springer, pp. 402-417, doi 10.1007/978-3-642-39634-2_29.

[18] Steven Schäfer, Gert Smolka \& Tobias Tebbi (2015): Completeness and Decidability of de Bruijn Substitution Algebra in Coq. In Xavier Leroy \& Alwen Tiu, editors: Proceedings of the 2015 Conference on Certified Programs and Proofs, CPP 2015, Mumbai, India, January 15-17, 2015, ACM, pp. 67-73, doi: $10.1145 / 2676724.2693163$

[19] Steven Schäfer, Tobias Tebbi \& Gert Smolka (2015): Autosubst: Reasoning with de Bruijn Terms and Parallel Substitutions. In Christian Urban \& Xingyuan Zhang, editors: Interactive Theorem Proving - 6th International Conference, ITP 2015, Nanjing, China, August 24-27, 2015, Proceedings, Lecture Notes in Computer Science 9236, Springer, pp. 359-374, doi:10.1007/978-3-319-22102-1_24.

[20] Kathrin Stark, Steven Schäfer \& Jonas Kaiser (2019): Autosubst 2: reasoning with multi-sorted de Bruijn terms and vector substitutions. In Assia Mahboubi \& Magnus O. Myreen, editors: Proceedings of the 8th ACM SIGPLAN International Conference on Certified Programs and Proofs, CPP 2019, Cascais, Portugal, January 14-15, 2019, ACM, pp. 166-180, doi 10.1145/3293880.3294101.

[21] Christian Sternagel \& René Thiemann (2015): Deriving class instances for datatypes. Arch. Formal Proofs 2015. Available at https://www.isa-afp.org/entries/Deriving.shtml. 